\title{
Diversity-Multiplexing Tradeoff Bounds for Wireless Relay Networks
}

\author{
John Boyer, David D. Falconer, Halim Yanikomeroglu \\ Broadband Communications and Wireless Systems Centre \\ Department of Systems and Computer Engineering \\ Carleton University, Ottawa, Canada \\ \{jboyer, ddf, halim\}@ sce.carleton.ca
}

\begin{abstract}
This paper derives bounds on the diversitymultiplexing tradeoff of wireless relay networks with arbitrary link connectivity between cooperating terminals. The derived bounds are applicable when there are single and multiple antennas per terminal. Two classes of relaying method are analyzed, those requiring all cooperating terminals to correctly decode the transmitted signal in order for the destination to correctly decode (comprehensive decoding), and those requiring only a subset of cooperating terminals to correctly decode the transmitted signal in order for the destination to correctly decode (destination decoding). It is shown that the diversitymultiplexing tradeoff of comprehensive decoding is constrained by the minimum number and arrangement of incident interterminal antenna links across all receiving terminals in the network, and that the diversity-multiplexing tradeoff of destination decoding is constrained by the minimum number and arrangement of incident inter-terminal antenna links across all cut sets in the network. Results for the complexity of the involved minimization algorithms are provided.
\end{abstract}

Index Terms - cooperative diversity, cooperative relaying, diversity-multiplexing tradeoff, relay networks, spatial diversity

\section{INTRODUCTION}

The spatial redundancy introduced by the presence of multiple antennas in wireless relay networks can in theory be used either to increase the diversity gain for a particular data rate or to increase the multiplexing gain (data rate) for a particular diversity gain. The concept of diversitymultiplexing tradeoff that was introduced in [11] formalizes this fundamental tradeoff between diversity and multiplexing gain. This paper derives bounds on the diversity-multiplexing tradeoff of wireless relay networks with any number of relay terminals and any possible combination of links between cooperating terminals, and extends the previously presented maximum diversity order results of [3].

Two classes of relaying method are considered, those requiring all cooperating terminals to correctly decode the transmitted information signal in order for the destination to correctly decode (comprehensive decoding), and those requiring only a subset of cooperating terminals to correctly decode the transmitted information signal in order for the destination terminal to correctly decode (destination decoding). For the destination decoding class, individual relays may attempt to decode the information signal before retransmission, but the key point is that only a subset are required to do so correctly in order for the destination to correctly decode.

The comprehensive decoding class includes decoded relaying with error propagation, also known as fixed decodeand-forward (DF) relaying. The destination decoding class includes decoded relaying without error propagation, also known as adaptive or selective decode-and-forward (DF) relaying, and amplified relaying, also known as amplify-andforward (AF) relaying. From the perspective of diversity order, this classification of comprehensive decoding versus destination decoding is more fundamental than that of decode-and-forward versus amplify-and-forward relaying. As noted in many existing publications such as [2], [3], [4], [7], and [8], the maximum achievable diversity order of wireless relay networks is not dependent on the physical layer relaying method (amplified or decoded), but instead on the criteria for retransmission at each relay and the presence of error propagation. The approach taken in this paper of deriving sometimes looser diversity-multiplexing tradeoff bounds for the most general wireless relay networks with multiple antennas is different than that of [10], where tighter diversitymultiplexing tradeoff bounds are derived, but only for specific simpler sub-problems of the most general wireless relay networks.

\section{SYSTEM MODEL}

The system model considered in this paper for a single user transmission in a wireless relay network involves a source terminal, a destination terminal, and a variable number of intermediate relay terminals. Let $T_{R}$ denote the set of all receiving terminals participating in a particular wireless relay network, let $S_{R}$ denote the complete set of distinct cut sets associated with the corresponding directed network graph, and let $L_{R}$ denote the complete set of inter-terminal antenna links associated with the corresponding directed network graph. Also, let $A_{i}$ denote the set of antennas at terminal $T_{i}$, and let $T_{P(i)}$ denote the set of previous terminals that transmit a signal received by terminal $T_{i}$. Finally, let $L_{i}$ denote the set of inter-terminal antenna links associated with a particular cut set $S_{i}$, and let $T_{I N(i)}$ and $T_{O U T(i)}$ respectively denote the sets of terminals on the input and output sides of cut set $S_{i}$ that are associated with at least one of the inter-terminal antenna links in the corresponding set $L_{i}$. Fig. 1 shows an example network, annotated with this terminology.

This defined set notation is used in variable subscripts of the form $x_{T_{i}}$ to denote specific terminals, groups of terminals, or cut sets. Notation of the form $x_{T_{i}}$ is abbreviated to $x_{i}$ for simplicity of exposition. The cardinality of a set $X$ is denoted by $|X|$. Furthermore, it is assumed that all relays operate in half-duplex mode and that a network with $N$ transmitters in general requires $2 \leq K \leq N$ orthogonal channels, resulting in a rate factor of $1 / K$. The system model 
does not imply any particular method by which the set of relay terminals, or set of active links between the pairs of terminals, are chosen.

In general, is not possible to directly determine the diversity-multiplexing tradeoff of wireless networks with arbitrary link connectivity between cooperating terminals from the high SNR probability of outage and maximum diversity order results presented in [3]. This is due to the fact that the terminals (for comprehensive decoding), or cut sets (for destination decoding), that limit the maximum achievable diversity order may not necessarily be the same terminals, or cut sets, that limit the maximum achievable multiplexing gain. The key difference is that although the maximum achievable diversity order depends on the number of independent fading realizations that can be combined, the maximum achievable multiplexing gain instead depends on the number of degrees of freedom in the channel. The terminal, or cut set, with the minimum number of independent fading realizations is not necessarily the terminal, or cut set, with the minimum number of degrees of freedom across all terminals, or cut sets, in the network.

Instead, the method used in this paper to bound the diversity-multiplexing tradeoff of wireless relay networks with arbitrary link connectivity between cooperating terminals applies the main result of [11] where it is shown that the diversity-multiplexing tradeoff curve of a multipleinput multiple-output (MIMO) channel with $m$ transmit antennas, $n$ receive antennas, and independent identically distributed Rayleigh fading between each pair of antennas is given by the piecewise linear function connecting the points $(r, d(r)), r=0,1, \ldots, \min \{m, n\}$, where $d(r)=(m-r)(n-r)$, $d(r)$ is the diversity order, and $r$ is the multiplexing gain.

We note that although this result was generated for MIMO channels, it is equivalent to the MISO/SIMO diversitymultiplexing tradeoff curve of $d(r)=m n(1-r)$ for the relevant range $r=0,1$. The method of bounding and minimization across cut sets used in this paper is similar to that applied independently in [9] and [10]. Different from [1], for practicality the model is constrained to non-overlapping symbol periods.

\section{NETWORKS WITH COMPREHENSIVE DECODING}

For networks with comprehensive decoding the diversitymultiplexing tradeoff curve can be upper bounded by performing a minimization across the diversity-multiplexing tradeoff curves of all terminals in the network. Applying the main result of [11], the diversity-multiplexing tradeoff curve of the channel at terminal $T_{i}$ (between terminal $T_{i}$ and all immediately preceding terminals $T_{P(i)}$ ) is given by the piecewise linear function connecting the points

$$
\begin{gathered}
(r, d(r)), r=0,1 / K, \ldots, \min \left\{\sum_{\left.T_{k} \in T_{P(i)}\left|A_{k}\right|,\left|A_{i}\right|\right\} / K, \text { where }}\right. \\
d(r)=\left(\sum_{T_{k} \in T_{P(i)}}\left|A_{k}\right|-K r\right)\left(\left|A_{i}\right|-K r\right) .
\end{gathered}
$$

This result can be applied across all terminals in the network, such that for networks with comprehensive decoding the diversity-multiplexing tradeoff curve is upper bounded by the piecewise linear function connecting the

points

$$
(r, d(r)), r=0,1 / K, \ldots, \min _{T_{i} \in T_{R}}\left\{\min \left\{\sum_{T_{k} \in T_{P(i)}}\left|A_{k}\right|,\left|A_{i}\right|\right\}\right\} / K,
$$

where

$$
d(r) \leq \min _{T_{i} \in T_{R}}\left\{\left(\sum_{T_{k} \in T_{P(i)}}\left|A_{k}\right|-K r\right)\left(\left|A_{i}\right|-K r\right)\right\} .
$$

From the diversity-multiplexing tradeoff curve it can be seen that the maximum achievable diversity order is given by

$$
d_{\max }=\min _{T_{i} \in T_{R}}\left\{\sum_{T_{k} \in T_{P(i)}}\left|A_{k} \| A_{i}\right|\right\},
$$

the minimum number of incident inter-terminal antenna links across all terminals, and the maximum achievable multiplexing gain is given by

$$
r_{\max }=\min _{T_{i} \in T_{R}}\left\{\sum_{T_{k} \in T_{P(i)}}\left|A_{k}\right|,\left|A_{i}\right|\right\} / K,
$$

the minimum number of transmit or receive antennas for the incident multiple antenna channels across all terminals, divided by the number of orthogonal channels required to operate the given transmission scheme.

These results indicate that the diversity-multiplexing tradeoff of comprehensive decoding is constrained by the minimum number and arrangement of incident inter-terminal antenna links across all receiving terminals in the network. Fig. 2 shows an example network, indicating those terminals with more than one antenna and annotated with the achievable diversity order of each terminal. Fig. 3 shows an example network, indicating those terminals with more than one antenna and annotated with the achievable multiplexing gain (not including the rate factor $1 / K$ ) of each terminal.

\section{NETwOrKS With DESTINATION DECODING}

For networks with destination decoding the diversitymultiplexing tradeoff curve can be upper bounded by performing a minimization across the diversity-multiplexing tradeoff curves of all cut sets in the network. Applying the main result of [11], the diversity-multiplexing tradeoff curve of the channel at cut set $S_{i}$ (across all the inter-terminal antenna links associated with cut set $S_{i}$ ) is upper bounded by the piecewise linear function connecting the points

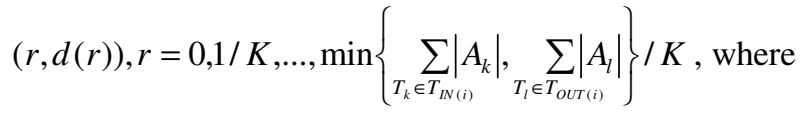

$$
\begin{aligned}
& d(r) \leq\left(\sum_{T_{k} \in T_{I N(i)}}\left|A_{k}\right|-K r\right)\left(\sum_{T_{l} \in T_{\text {OUT }(i)}}\left|A_{l}\right|-K r\right),
\end{aligned}
$$

where the upper bound results from the fact that the sets of terminals on the input and output sides of cut set $S_{i}, T_{I N(i)}$ and $T_{\text {OUT (i) }}$ respectively, are not necessarily fully connected. For example, in the network shown in Fig. 4, for the cut set $S_{4}$ where terminals $T_{S}, T_{R 1}, T_{R 2}$, and $T_{R 3}$ belong to $T_{I N(4)}$ and terminals $T_{R 4}, T_{R 5}$, and $T_{D}$ belong to $T_{\text {OUT (4) }}$, although 
terminal $T_{R 3}$ is directly connected to terminals $T_{R 4}$ and $T_{D}$, it is not directly connected to terminal $T_{R 5}$. This reduction in connectivity in comparison to a fully connected MIMO channel between the sets of terminals on the input and output sides of cut set $S_{4}$ means that the diversity-multiplexing tradeoff of that corresponding fully connected MIMO channel is an upper bound on the actual diversitymultiplexing tradeoff of the channel at cut set $S_{4}$. In general, the diversity-multiplexing tradeoff of the channel at cut set $S_{i}$ is upper bounded by the diversity-multiplexing tradeoff of the corresponding fully connected MIMO channel involving the same sets of terminals on the input and output sides of cut set $S_{i}$. This upper bound is tight when the sets of terminals on the input and output sides of cut set $S_{i}$ are fully connected.

For networks with destination decoding the diversitymultiplexing tradeoff is therefore upper bounded by the piecewise linear function connecting the points

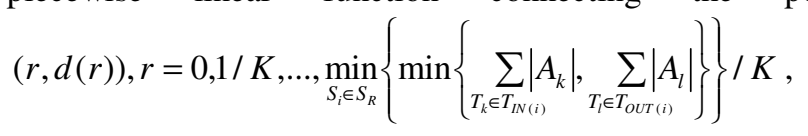

where

$$
d(r) \leq \min _{S_{i} \in S_{R}}\left\{\left(\sum_{T_{k} \in T_{\text {IN }(i)}}\left|A_{k}\right|-K r\right)\left(\sum_{T_{l} \in T_{\text {OUT }(i)}}\left|A_{l}\right|-K r\right)\right\} .
$$

This upper bound is tight when the sets of terminals on the input and output sides of all cut sets are fully connected.

From the diversity-multiplexing tradeoff curve it can be seen that the maximum achievable diversity order is upper bounded by

$$
d_{\max } \leq \min _{S_{i} \in S_{R}}\left\{\sum_{T_{k} \in T_{I N(i)}}\left|A_{k}\right| \sum_{T_{l} \in T_{\text {OUT }(i)}}\left|A_{l}\right|\right\},
$$

the minimum number of inter-terminal antenna links across all cut sets when the sets of terminals on the input and output sides of all cut sets are fully connected, and the maximum achievable multiplexing gain is upper bounded by

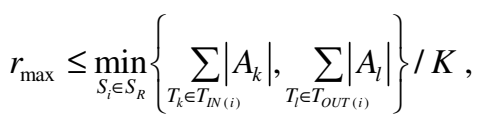

the minimum number of transmit or receive antennas for the incident multiple antenna channels across all cut sets, divided by the number of orthogonal channels required to operate the given transmission scheme.

We now consider a refinement that provides a more precise result than the upper bound of (5). As noted in [5] and [11], the diversity-multiplexing tradeoff of a MIMO channel degrades when the channel matrix is rank deficient. It is shown in [5] that the diversity-multiplexing tradeoff curve of a rank deficient MIMO channel with $m$ transmit antennas, $n$ receive antennas, and independent identically distributed Rayleigh fading between each pair of antennas is given by the piecewise linear function connecting the points $(r, d(r)), r=0,1, \ldots, \kappa$, where $\kappa \leq \min \{m, n\}$ is the rank of the MIMO channel matrix and $d(r)=(m+n-\kappa-r)(\kappa-r)$.
Since the fact that the sets of terminals on the input and output sides of cut set $S_{i}$ are not fully connected may result in the corresponding MIMO channel being rank deficient, the diversity-multiplexing tradeoff curve of the channel at cut set $S_{i}$ is given by the piecewise linear function connecting the points $(r, d(r)), r=0,1 / K, \ldots, \kappa_{i} / K$, where

$$
d(r)=\left(\sum_{T_{k} \in T_{I N(i)}}\left|A_{k}\right|+\sum_{T_{l} \in T_{\text {OUT }(i)}}\left|A_{l}\right|-\kappa_{i}-K r\right)\left(\kappa_{i}-K r\right),
$$

and $\kappa_{i} \leq \min \left\{\sum_{T_{k} \in T_{I N(i)}}\left|A_{k}\right|, \sum_{T_{i} \in T_{\text {OUT }(i)}}\left|A_{l}\right|\right\} \quad$ is the rank of the corresponding MIMO channel matrix at cut set $S_{i}$. From this diversity-multiplexing tradeoff curve it can be seen that the maximum achievable diversity order at cut set $S_{i}$ is given by

$$
d_{\max }=\left(\sum_{T_{k} \in T_{I N(i)}}\left|A_{k}\right|+\sum_{T_{l} \in T_{\text {OUT }(i)}}\left|A_{l}\right|-\kappa_{i}\right) \kappa_{i} .
$$

However, it was previously indicated in [3] that the maximum achievable diversity order at cut set $S_{i}$ is $d_{\max }=\left|L_{i}\right|$, so it is clear that

$$
\left(\sum_{T_{k} \in T_{I N(i)}}\left|A_{k}\right|+\sum_{T_{l} \in T_{\text {OUT }(i)}}\left|A_{l}\right|-\kappa_{i}\right) \kappa_{i}=\left|L_{i}\right| .
$$

Therefore, the diversity-multiplexing tradeoff curve at cut set $S_{i}$ can be rewritten as

$$
\begin{aligned}
& d(r)=\left(\sum_{T_{k} \in T_{I N(i)}}\left|A_{k}\right|+\sum_{T_{l} \in T_{\text {OUT }(i)}}\left|A_{l}\right|-\kappa_{i}-K r\right)\left(\kappa_{i}-K r\right) \\
& =\left(\sum_{T_{k} \in T_{\text {IN }(i)}}\left|A_{k}\right|+\sum_{T_{l} \in T_{\text {OUT }(i)}}\left|A_{l}\right|-\kappa_{i}\right) \kappa_{i}+(K r)^{2} \\
& -\left(\sum_{T_{k} \in T_{I N(i)}}\left|A_{k}\right|+\sum_{T_{l} \in T_{\text {OUT }(i)}}\left|A_{l}\right|\right) K r \\
& =\left|L_{i}\right|+(K r)^{2}-\left(\sum_{T_{k} \in T_{I N(i)}}\left|A_{k}\right|+\sum_{T_{l} \in T_{\text {OUT }(i)}}\left|A_{l}\right|\right) K r \\
& =\left|L_{i}\right|-\left(\sum_{T_{k} \in T_{I N(i)}}\left|A_{k}\right|+\sum_{T_{l} \in T_{\text {OUT }(i)}}\left|A_{l}\right|-K r\right) K r
\end{aligned}
$$

for $r=0,1 / K, \ldots, \kappa_{i} / K$. This result can be applied across all cut sets in the network, such that for networks with destination decoding the diversity-multiplexing tradeoff curve is upper bounded by the piecewise linear function connecting the points $(r, d(r)), r=0,1 / K, \ldots, \min _{S_{i} \in S_{R}}\left\{\kappa_{i}\right\} / K$, where

$$
\left.d(r) \leq \min _{S_{i} \in S_{R}}\left\{\left|L_{i}\right|-\left(\sum_{T_{k} \in T_{I N(i)}}\left|A_{k}\right|+\sum_{T_{i} \in T_{\text {OUT }(i)}}\left|A_{l}\right|-K r\right) K r\right)\right\} .
$$

From the diversity-multiplexing tradeoff curve it can be seen that the maximum achievable diversity order is given by

$$
d_{\max }=\min _{S_{i} \in S_{R}}\left\{\left|L_{i}\right|\right\}
$$

the minimum number of inter-terminal antenna links across all cut sets, and the maximum achievable multiplexing gain is given by

$$
r_{\max }=\min _{S_{i} \in S_{R}}\left\{\kappa_{i}\right\} / K
$$


the minimum rank of the channel matrix across all cut sets, divided by the number of orthogonal channels required to operate the given transmission scheme.

These results indicate that the diversity-multiplexing tradeoff of destination decoding is constrained by the minimum number and arrangement of incident inter-terminal antenna links across all cut sets in the network. Fig. 4 shows an example network, indicating those terminals with more than one antenna and annotated with the achievable diversity order of each cut set that is relevant given the directed connectivity. Fig. 5 shows an example network, indicating those terminals with more than one antenna and annotated with the achievable multiplexing gain (not including the rate factor $1 / K$ ) of each cut set that is relevant given the directed connectivity.

Comparing the diversity-multiplex tradeoff curves of (5) and (9) for networks with destination decoding, it can be seen that the rank deficiency of the channel at cut set $S_{i}$ degrades the corresponding diversity-multiplexing tradeoff curve in comparison to fully connected MIMO channels (when the sets of terminals on the input and output sides of all cut sets are fully connected). The diversity order degradation at cut set $S_{i}$ is given by

$$
\begin{aligned}
& \Delta d_{i}=\left(\sum_{T_{k} \in T_{\text {IN }(i)}}\left|A_{k}\right|-K r\right)\left(\sum_{T_{l} \in T_{\text {OUT }(i)}}\left|A_{l}\right|-K r\right) \\
& -\left(\sum_{T_{k} \in T_{I N(i)}}\left|A_{k}\right|+\sum_{T_{i} \in T_{\text {OUT }(i)}}\left|A_{l}\right|-\kappa_{i}-K r\right)\left(\kappa_{i}-K r\right), \\
& =\left(\sum_{T_{k} \in T_{I N(i)}}\left|A_{k}\right|-\kappa_{i}\right)\left(\sum_{T_{i} \in T_{\text {OUT }(i)}}\left|A_{l}\right|-\kappa_{i}\right)
\end{aligned}
$$

which we note is independent of the rate $r$. Considering the maximum achievable diversity order corresponding to (5) and (9), the diversity order degradation at cut set $S_{i}$ is also given by

$$
\Delta d_{i}=\left(\sum_{T_{k} \in T_{I N(i)}}\left|A_{k}\right| \sum_{T_{i} \in T_{\text {OUT }(i)}}\left|A_{l}\right|\right)-\left|L_{i}\right|
$$

and comparing the results of (7) and (14), the diversity order degradation for wireless relay networks with destination decoding is given by

$$
\Delta d=\min _{S_{i} \in S_{R}}\left\{\sum_{T_{k} \in T_{I N(i)}}\left|A_{k}\right| \sum_{T_{l} \in T_{\text {OUT }(i)}}\left|A_{l}\right|\right\}-\min _{S_{i} \in S_{R}}\left\{\left|L_{i}\right|\right\} .
$$

Considering the maximum achievable multiplexing gain corresponding to (5) and (9), the maximum multiplexing gain degradation at cut set $S_{i}$ is given by

$$
\Delta r_{\max , i}=\min \left\{\sum_{T_{k} \in T_{\text {IN }(i)}}\left|A_{k}\right|-\kappa_{i}, \sum_{T_{i} \in T_{\text {OUT }(i)}}\left|A_{l}\right|-\kappa_{i}\right\} / K
$$

and comparing the results of (8) and (15), the maximum multiplexing gain degradation for wireless relay networks with destination decoding is given by

$$
\Delta r_{\max }=\min _{S_{i} \in S_{R}}\left\{\sum_{T_{k} \in T_{\text {IN }(i)}}\left|A_{k}\right|, \sum_{T_{l} \in T_{\text {OUT }(i)}}\left|A_{l}\right|\right\} / K-\min _{S_{i} \in S_{R}}\left\{\kappa_{i}\right\} / K,
$$

\section{COMPLEXITY OF Minimization AlgORITHMS}

In this paper we have repeatedly used results that include a minimization of diversity-multiplexing tradeoff across all terminals or cut sets in a wireless relay network. It is therefore very relevant to consider the algorithmic complexity of this minimization process for the parameters and relaying method classes of interest. We now consider the complexity of the relevant algorithms for minimization across all terminals or cut sets in the network. We use the O-notation presented in [6], according to the following definition, to describe the asymptotic complexity of a function $f(n)$ with respect to $n$. Let $f(n)$ and $g(n)$ be two functions defined on some subset of the real numbers. Then $f(n) \in O(g(n))$ as $n \rightarrow \infty$ if an only if $\exists n_{0}, \exists M>0,|f(n)| \leq M|g(n)|$ for $n>n_{0}$. This can be read that $f(n)$ is of the order of $g(n)$.

The number of possible cut sets in a wireless relay network with $N$ terminals is given by

$$
\begin{aligned}
\left|S_{R}\right| & =\sum_{i=0}^{N-2} \frac{(N-2) !}{(N-2-i) ! i !}, \\
& =2^{N-2}
\end{aligned}
$$

where the reason that this is a function of $N-2$ instead of $N$ is that the source and destination terminals are always on the source and sink sides of all cut sets respectively.

When performing a minimization of the diversitymultiplexing tradeoff across all terminals, respectively cut sets, in a network there is a first comparison cycle to calculate the value of the minimum number of degrees of freedom in the channel across all terminals $F_{T}$, respectively the minimum number of degrees of freedom in the channel across all cut sets $F_{S}$, and then a subsequent $F_{T}$, respectively $F_{S}$, comparison cycles to perform the minimization for each integer value of the multiplexing gain $r \leq F_{T}$, respectively $r \leq F_{S}$. The minimum number of degrees of freedom in the channel across all terminals in the network is given by

$$
F_{T}=\min _{T_{i} \in T_{R}}\left\{\min \left\{\sum_{T_{k} \in T_{P(i)}}\left|A_{k}\right|,\left|A_{i}\right|\right\}\right\},
$$

and the minimum number of degrees of freedom in the channel across all cut sets in the network is given by

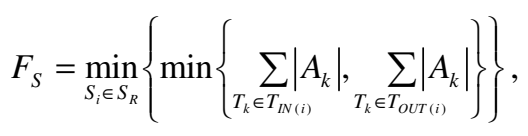

assuming the corresponding channel matrices are full rank.

Calculating the complexity of diversity-multiplexing tradeoff minimization across all terminals for networks with comprehensive decoding is straightforward. The algorithm includes $(N-1)\left(F_{T}+1\right)$ comparisons between terminals. The complexity of algorithms for minimization of the diversitymultiplexing tradeoff across all terminals in a network with $N$ terminals is therefore $(N-1)\left(F_{T}+1\right) \in O(N)$. Similarly, the baseline complexity of diversity-multiplexing tradeoff minimization across all cut sets for networks with destination 
decoding is an algorithm that brute-force searches across all cut sets. The algorithm includes $\left(2^{N-2}-1\right)\left(F_{S}+1\right)$ comparisons between cut sets. The complexity of algorithms for minimization of the diversity-multiplexing tradeoff across all cut sets in a network with $N$ terminals is therefore upper bounded by $\left(2^{N-2}-1\right)\left(F_{S}+1\right) \in O\left(2^{N}\right)$.

The brute-force algorithm of minimization across all cut sets in a network is exponential in complexity and therefore not very practical for large networks. However, a number of more efficient algorithms for determining the maximum flow of a directed network graph have been developed and are reported in [6]. These can be applied in a more efficient manner for the minimization of the diversity-multiplexing tradeoff across all cut sets with an intelligent choice of edge weights applied to the inter-terminal antenna links. The FordFulkerson algorithm [6] has a complexity of $O\left(\left|L_{R}\right| d_{\max }\right)$. The Edmonds-Karp algorithm [6] has a complexity of $O\left(N\left|L_{R}\right|^{2}\right)$. The Relabel-to-Front algorithm [6] has a complexity of $O\left(N^{3}\right)$. The theoretically minimum complexity algorithm for determining the maximum flow of a directed network graph has a complexity of $O\left(N\left|L_{R}\right|\right)$, but so far no practical algorithms has been developed that achieve that complexity [6]. In large networks where the number of terminals and inter-terminal links is large, the Relabel-toFront algorithm will generally have the lowest complexity of existing practical algorithms, so a complexity of $O\left(N^{3}\right)$ for minimization across all cut sets in a network with $N$ terminals is a reasonable practical baseline.

\section{CONCLUDING REMARKS}

This paper has derived bounds on the diversitymultiplexing tradeoff of wireless relay networks with arbitrary link connectivity between cooperating terminals. When all cooperating terminals must correctly decode in order for the destination terminal to correctly decode (comprehensive decoding) it is shown that the diversitymultiplexing tradeoff is constrained by the minimum number and arrangement of incident inter-terminal antenna links across all receiving terminals in the network. When only a subset of cooperating terminals must correctly decode in order for the destination terminal to correctly decode (destination decoding) it is shown that the diversitymultiplexing tradeoff is constrained by the minimum number and arrangement of associated inter-terminal antenna links across all cut sets in the network.

It is also noted that the terminals (for comprehensive decoding), or cut sets (for destination decoding), that limit the maximum achievable diversity order may not necessarily be the same terminals, or cut sets, that limit the maximum achievable multiplexing gain. The terminal, or cut set, with the minimum number of independent fading realizations is not necessarily the terminal, or cut set, with the minimum number of degrees of freedom across all terminals, or cut sets, in the network. This means that the method of minimization across terminals or cut sets must be performed independently for each possible value of the multiplexing gain in the diversity-multiplex tradeoff curve. The complexity of practical algorithms for minimization of the diversitymultiplexing tradeoff across all terminals in a network with comprehensive decoding and $N$ terminals is $O(N)$. The complexity of practical algorithms for minimization of the diversity-multiplexing tradeoff across all cut sets in a network with destination decoding and $N$ terminals is $O\left(N^{3}\right)$.

\section{REFERENCES}

[1] K. Azarian, H. Gamal, and P. Schniter, "On the achievable diversitymultiplexing tradeoff in half-duplex cooperative channels," IEEE Trans. on Information Theory, vol. 51, no. 12, pp. 4152-4172, December 2005.

[2] J. Boyer, D. D. Falconer, and H. Yanikomeroglu, "Multihop diversity in wireless relaying channels," IEEE Trans. on Communications, vol. 52, no. 10, pp. 1820-1830, October 2004.

[3] J. Boyer, D. Falconer, and H. Yanikomeroglu, "Diversity order bounds for wireless relay networks," Proc. of IEEE Wireless Communications and Networking Conference, pp. 1800-1804, March 2007.

[4] J. Boyer, D. Falconer, and H. Yanikomeroglu, "Cooperative connectivity models for wireless relay networks," IEEE Trans. on Wireless Communications, vol. 6, no. 6, pp. 1992-2000, June 2007.

[5] W. Chang, S. Chung, and Y. Lee, "Diversity-multiplexing tradeoff in rank-deficient and spatially correlated MIMO channels," Proc. of IEEE International Symposium on Information Theory, pp. 1144-1148, July 2006.

[6] T. Cormen, C. Leiserson, R. Rivest, and C. Stein, Introduction to Algorithms, McGraw-Hill, Inc., New York, Second edition, 2001.

[7] J. Nicholas Laneman and G. Wornell, "Distributed space-time-code protocols for exploiting cooperative diversity in wireless networks," IEEE Trans. on Information Theory, vol. 49, no. 10, pp. 2415-2425, October 2003.

[8] J. Nicholas Laneman, "Limiting analysis of outage probabilities for diversity schemes in fading channels," IEEE Global Telecommunications Conference, vol. 3, pp. 1242-1246, December 2003.

[9] M. Yuksel and E. Erkip, "Diversity-multiplexing tradeoff in halfduplex relay systems," Proc. of IEEE International Conference on Communications, June 2007.

[10] M. Yuksel and E. Erkip, "Multi-antenna cooperative wireless systems: A diversity multiplexing tradeoff perspective," IEEE Trans. on Information Theory, Special Issue on Models, Theory, and Codes for Relaying and Cooperation in Communication Networks, vol. 53, no. 10, pp. 3371-3393, October 2007.

[11] L. Zheng and D. Tse, "Diversity and multiplexing: A fundamental tradeoff in multiple-antenna channels," IEEE Trans. on Information Theory, vol. 49, no. 5, pp. 1073-1096, May 2003.

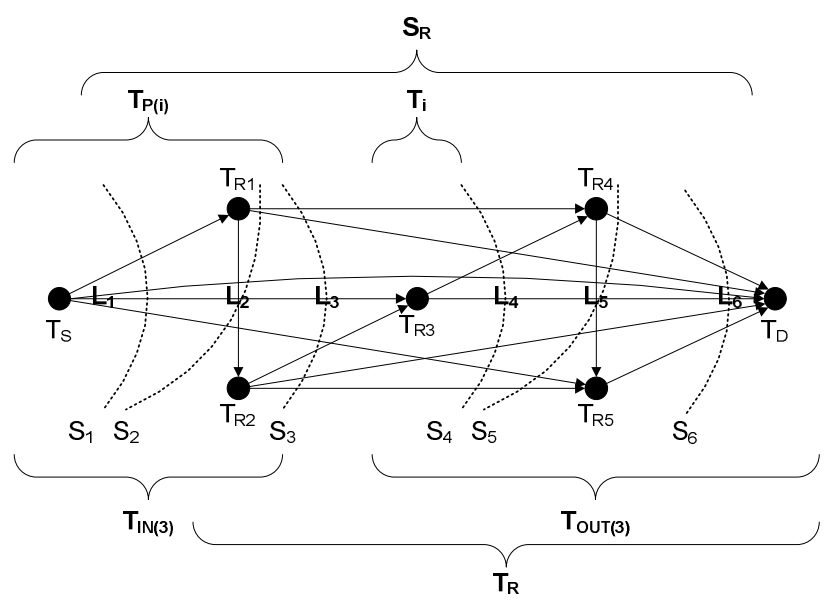

Fig. 1. Wireless Relay Network Terminology 


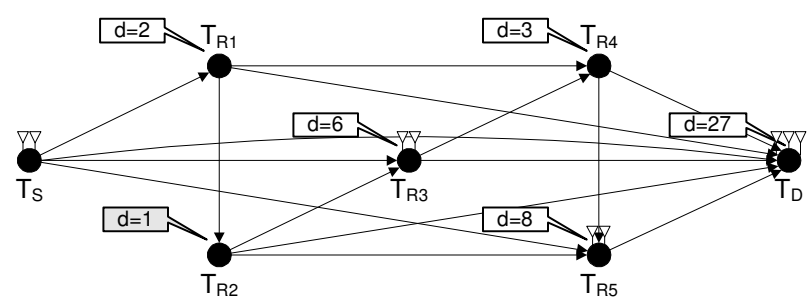

Fig. 2. Example: Diversity Order with Comprehensive Decoding

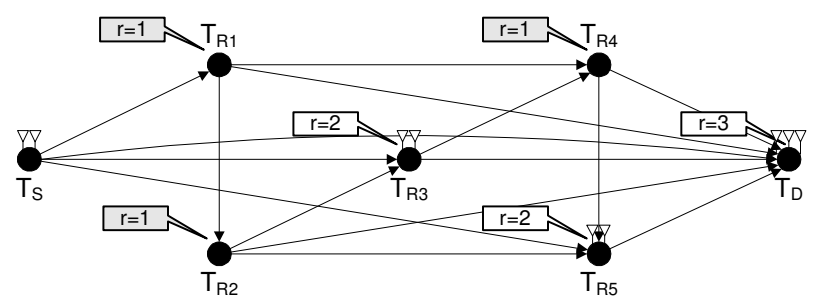

Fig. 3. Example: Multiplexing Gain with Comprehensive Decoding

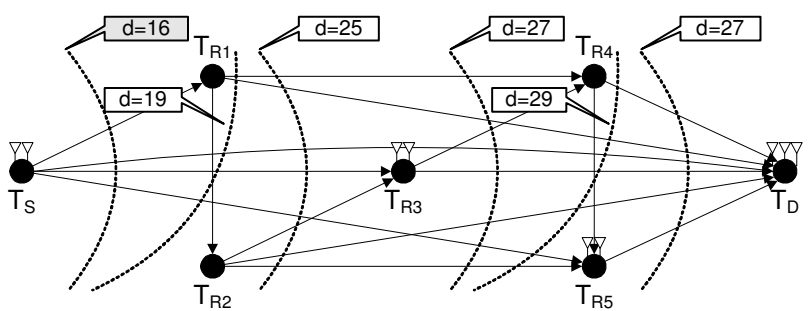

Fig. 4. Example: Diversity Order with Destination Decoding

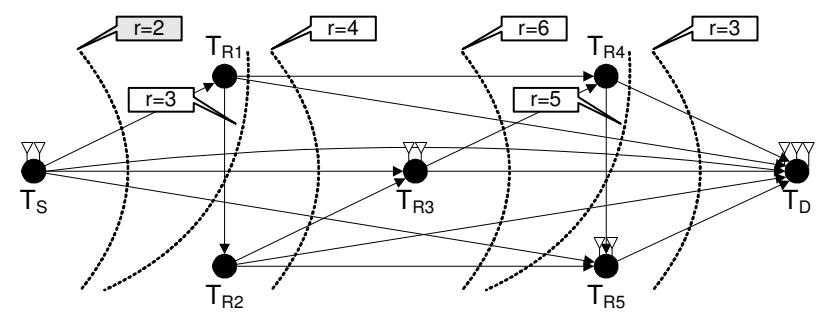

Fig. 5. Example: Multiplexing Gain with Destination Decoding 